\title{
Efecto de Intervención Innovadora: Consejería de Enfermería en Salud Cardiovascular en Atención Primaria
}

Vivian Vilchez, Tatiana Paravic, Alide Salazar, Katia Sáez.

\author{
1. Institución: Programa de Salud Cardiovascular de los seis Centros de \\ Salud Familiar (CESFAM) Municipalizados de Concepción. \\ Apoyo financiero de: \\ - Universidad de Costa Rica. \\ - Vicerrectoría de Investigación, Universidad de Concepción. \\ - Dirección de Postgrado, Universidad de Concepción. \\ - Convenio de Desempeño UCO1201, Universidad de Concepción. \\ - INNOVA-BIO BIO: Línea para apoyar la realización de Tesis de Postgrado y \\ 11ava Convocatoria Innovación Emprendedora.
}

Antecedentes: La prevención de enfermedades cardiovasculares es un reto para los sistemas de salud. Objetivo: Establecer el efecto de una intervención innovadora de Consejería de Enfermería Personalizada, Presencial y Telefónica, dirigida a: control de factores de riesgo cardiovascular (hipertensión, dislipidemia, sobrepeso), mejoramiento de calidad de vida relacionada con salud (CVRS), fortaleciendo la autoeficacia, el apoyo social y estructurando el fenómeno "vivir con expectativas de cambio" en personas usuarias del programa de salud cardiovascular de los Centros de Salud Municipales de Concepción.

Método: Se usó método mixto anidado, como método primario el cuantitativo, ensayo clínico controlado aleatorizado. Se randomizaron controles $(\mathrm{n}=56)$ e intervenidos $(n=53)$ sometidos a 10 sesiones presenciales y 5 telefónicas. Se midió CVRS, presión arterial, IMC, circunferencia abdominal, colesterol total, LDL, HDL, LDL/HDL), CT/HDL y Riesgo Coronario a 10 años. El método secundario cualitativo fue el de Parse. fenomenológico-hermenéutico. Resultados: mujeres y hombres disminuyeron circunferencia abdominal, colesterol total, colesterol-LDL, CT/HDL $(\mathrm{p}<0,05)$; las mujeres disminuyeron significativamente peso y aumentaron CVRS. Los hombres disminuyeron presión arterial sistólica y diastólica, LDL/HDL, y riesgo coronario a 10 años. En lo cualitativo se estructura "vivir con expectativas de cambio", como un proceso interactivo de cuidado que integran lo físico y psicológico, el cual establece alianzas que generan compromisos personales y contribuyen a enfrentar las dificultades con perseverancia y con acciones innovadoras.

Conclusiones: Esta intervención es una estrategia efectiva para el control de tres factores de riesgo cardiovascular y mejoramiento de CVRS. 


\section{Effect of an innovative process of nursing counseling on cardiovascular health in a primary health care setting}

Background: prevention of cardiovascular disease remains a challenge for health care systems

Aim: to evaluate the effect of an innovative process of personalized nursing counseling including telephone monitoring on cardiovascular risk factors and quality of life indexes.

Method: the intervention took place at municipal health care facilities involved in the program for cardiovascular health in Concepción, Chile. After randomization, 56 subjects acted as controls and 53 were submitted to 10 personalized and 5 telephone counseling sessions. Variables measured included CVRS, blood pressure, pressure, BMI, abdominal circumference, total, LDL and HDL cholesterol levees and derived risk indexes. Coronary risk at 10 years was estimated. A parallel qualitative evaluation was performed through a phenomenological-hermeneutic method.

Results: abdominal circumference, total and LDL cholesterol and Chol/HDL ratio decreased in both males and females. Weight decreased and CVRS increased in females. Systolic and diastolic blood pressure, LDL/HDL ratio and 8-year risk of CVD decreased in males. Qualitative intervention was effective in developing an attitude of "living with an expectation of change" and in creating personal interactions to strengthen such attitude.

Conclusion: this innovative health intervention strategy was effective in the control of three cardiovascular risk factors and in improving CVRS

Key Words: Cardiovascular nursing, risk factors, quality of life, personalized counseling. 


\section{Introducción}

Las enfermedades cardiovasculares figuran como las principales Enfermedades No Transmisibles (ENT) a nivel mundial $^{1}$. En Chile, la enfermedad isquémica del corazón y la enfermedad cerebrovascular ${ }^{2}$ ocupan la primera causa de mortalidad, teniendo como factores de riesgo en común la hipertensión arterial, la dislipidemia y el estilo de vida. A pesar de la contribución del Programa de Salud Cardiovascular $(\mathrm{PSCV})^{3}$, aún prevalecen dichas enfermedades y continúan en aumento los factores de riesgo cardiovascular ${ }^{4}$. Por tal motivo, la prevención de los factores de riesgo cardiovascular y las complicaciones tardías que estos generan, son una de las tareas más importantes y complejas que enfrenta el sistema de salud ${ }^{4}$. La Organización Panamericana indica que se puede prevenir hasta el $75 \%$ de la enfermedad isquémica del corazón y de los accidentes cerebrovasculares, si se gestiona el conocimiento científico disponible sobre la prevención y el control de sus factores de riesgo, por medio de intervenciones costo-efectivas y asequibles que incorporen las vivencias de las personas que los presentan ${ }^{5}$. La evidencia señala la efectividad de intervenciones de Enfermería, dirigidas al control de factores de riesgo cardiovascular que combinan la metodología presencial con la telefónica ${ }^{6}$. Asimismo, muestran la responsabilidad del enfermero/a en la implementación de estrategias $^{7}$ en los grupos de intervención que producen mejoras significativas en indicadores de Presión Arterial, colesterol, Índice de Masa Corporal, actividad física y alimentación ${ }^{8}$. La utilización de teorías o modelos de Enfermería brinda los conocimientos y orientación requerida para que las/os Enfermeras/os descubran significados sobre las experiencias humanas de salud y calidad de vida de los usuarios ${ }^{9}$, mejorando la gestión del cuidado. El objetivo de esta experiencia fue establecer el efecto de una intervención innovadora de Consejería de Enfermería Personalizada, Presencial y Telefónica, dirigida a: control de factores de riesgo cardiovascular (hipertensión, dislipidemia, sobrepeso), mejoramiento de calidad de vida relacionada con salud (CVRS), fortaleciendo la autoeficacia, el apoyo social y estructurando el fenómeno "vivir con expectativas de cambio" en personas usuarias del programa de salud cardiovascular de los Centros de Salud Municipales de Concepción.

\section{Métodos}

Diseño mixto incrustado o anidado, compuesto por método cuantitativo (primario) y cualitativo (secundario), siguiendo criterio de complementariedad ${ }^{10}$, con la estrategia de integración en el momento de analizar e interpretar los datos.
Método Cuantitativo: Ensayo clínico controlado aleatorizado. Población de 640 personas entre 35 y 64 años inscritos en alguno de los 6 Centros de Salud Familiar Municipalizados de Concepción (CESFAM) en el PSCV, con tres factores de riesgo.

Criterios de inclusión: edad entre 35 y 64 años; ser portador de tres factores de riesgo cardiovascular (hipertensión, sobrepeso y dislipidemia); residir en el sector en el que está inscrito y firmar consentimiento informado. Criterios de exclusión: personas con diabetes, síndrome metabólico, cardiopatía isquémica, accidente cerebrovascular con secuelas, enfermedad pulmonar obstructiva crónica, alguna enfermedad mental o demencia, alcoholismo, enfermedades terminales, inmovilizadas, enfermedades tiroideas, cáncer, virus de inmunodeficiencia humana/sida, enfermedad reumática severa. Un total de 224 individuos cumplieron con los criterios de inclusión, 120 aceptaron participar en la investigación y firmaron el consentimiento informado. Seguidamente, se realizó pre-test.

Randomización: la conformación de grupos se realizó mediante análisis de conglomerados jerárquico utilizando la distancia de Mahalanobis y el algoritmo de Ward, considerando las variables de homologación. Se seleccionaron al azar 60 individuos para grupo control y 60 para ser intervenidos. Al aplicar los test respectivos, no se encontraron diferencias significativas entre ambos grupos. El Grupo Intervención, al final del estudio, quedó constituido por 53 personas y Grupo Control por 56. Ambos grupos continuaron recibiendo la atención tradicional otorgada por el PSCV del CESFAM.

Instrumentos: Cuestionario genérico de Calidad de Vida Relacionada con Salud SF-36v2 ${ }^{11}$, Escala de Autoeficacia General $^{12}$ y Escala Multidimensional Apoyo Social Percibido (MSPSS) ${ }^{13}$. Todos los instrumentos fueron previamente validados en Chile. Los indicadores de salud física que se midieron fueron los siguientes: el Índice de Masa Corporal $^{14}$ (IMC), Presión Arterial ${ }^{15}$ (PA), Circunferencia Abdominal $^{14}$ (CA) y el perfil lipídico incluyó: colesterol total (Col-Total), lipoproteínas de alta densidad (ColHDL), lipoproteínas de baja densidad (Col-LDL), triglicéridos, índice de aterogenicidad (LDL/HDL) y factor de riesgo cardiovascular (TC/HDL). El riesgo coronario a 10 años se calculó basado en las tablas de Framingham ${ }^{16}$.

Análisis estadístico: análisis de datos utilizando SAS Online Doc. versión 9.2 (SAS Institute Inc. Cary, NC, USA. 
2003) para estadística descriptiva e inferencial. Los datos fueron sometidos a análisis con los siguientes tests: Shapiro-Wilk, t de student, U de Mann-Whitney y Wilcoxon. Se utilizó un nivel de significancia $\alpha=0,05$.

Método Cualitativo: Método Parse ${ }^{17}$ fenomenológico-hermenéutico, diseñado para incrementar la comprensión de las experiencias de vida que propone la teoría Humanbecoming ${ }^{18}$. Es fenomenológico porque descubre la estructura de experiencias de vida descritas por participantes con el propósito de comprender el significado y es hermenéutico porque interpreta los hallazgos. Participaron 14 personas del grupo de intervención. Se estableció el diálogo en dos momentos: en la primera sesión de la Consejería se definieron las expectativas de cambio a trabajar y en la última sesión se dialogó sobre la experiencia de vivir con las expectativas de cambio fijadas al inicio del proceso de intervención. Los participantes fueron seleccionados por conveniencia y el número se definió a partir de la saturación de los resultados.

\section{Método en tres pasos ${ }^{17}$ :}

a) Intervención Dialógica: recolección de datos; los diálogos fueron grabados y transcritos palabra por palabra.

b) Extracción-Síntesis: Se extrajeron los significados de las experiencias de los participantes de vivir con expectativas de cambio.

c) Interpretación Heurística: dimensión interpretativa, enlazó el hallazgo del estudio con los principios de la teoría Humanbecoming ${ }^{17}$.

\begin{tabular}{|c|c|c|c|}
\hline Sesión & $P^{*}$ & $\mathrm{~T} \dagger$ & Temática \\
\hline 1 & $x$ & & Salud, CVRS y Expectativas de Cambio \\
\hline 2 & $x$ & & Menos Riesgo, Más Salud \\
\hline 3 & & & \\
\hline 4 & & & \\
\hline 5 & $x$ & $x$ & Hipertensión \\
\hline 6 & & & \\
\hline 7 & & & \\
\hline 8 & & $\mathrm{X}$ & Dislipidemia \\
\hline 9 & & & \\
\hline 10 & $x$ & & Comer Sano \\
\hline 11 & $x$ & & Salud Bucodental \\
\hline 12 & $x$ & & Movimiento \\
\hline 13 & $x$ & & Manejo del Estrés \\
\hline 14 & & & ¡Estoy Bien! \\
\hline 15 & $x$ & & $\begin{array}{l}\text { Haciendo un recorrido por las sesiones } \\
\text { recibidas y el impacto en CVRS }\end{array}$ \\
\hline
\end{tabular}

*: Presencial †े:Telefónica
Intervención La Consejería tuvo como eje central la CVRS, la autoeficacia, el apoyo social y la teoría Humanbecoming. Se diseñó con base en las necesidades de salud de la población meta, incluyendo 15 sesiones: 10 presenciales (entre 45- 60 minutos) y 5 sesiones telefónicas (15 minutos), desarrolladas por las investigadoras ${ }^{19}$. Se muestra en la Tabla 1 la distribución de las sesiones.

La investigación fue aprobada por el Comité de Bioética de la Facultad de Medicina de la Universidad de Concepción, Chile, registro DIFM 042/2012 y autorizada por la Dirección de Administración de Salud de Concepción. Consideró requisitos éticos de E. Emmanuel. Fue realizada en la Unidad de Prevención de Obesidad y Sobrepeso (UPOS) de la Universidad de Concepción.

\section{Resultados}

En la Tabla 2 se observa que ambos grupos están conformados por un mayor porcentaje de mujeres, la mayoría se encuentra en el rango de edad entre 55-64 años, con enseñanza media y casada.

La Tabla 3 muestra que las mujeres del grupo intervención presentaron mejoras significativas con respecto al grupo control en: peso, circunferencia abdominal, Col-Total, colLDL e LDL/HDL, en las dos medidas resumen de CVRS: Componente de Salud Física y Mental; entre la medición pre y pos-test.

En las Tabla 4 se observa que los hombres del grupo intervención presentaron cambios significativos con respecto al grupo control, en: CA, Col-Total, col-LDL, TC/HDL, LDL/HDL, Riesgo Coronario a 10 años, presión sistólica y diastólica; entre las mediciones pre y pos-test. Hubo una mejora altamente significativa de CA en el grupo intervenido con respecto al grupo control en CA.

Con respecto a los resultados cualitativos, tres conceptos de expectativas de cambio emergieron del diálogo comprometido y el proceso de extracción-síntesis: A) Visibilizar la realidad de salud, integrando lo físico y psicológico, a través de un proceso interactivo de cuidado con acción comunicativa. B) Establecimiento de alianzas para generar compromisos personales y enfrentar divergencias. C) Esfuerzo y perseverancia para realizar acciones innovadoras en salud que permitan adaptarse a las condiciones de salud que se van presentando. La estructura de la experiencia de vivir con expectativas de cambio se planteó de la siguiente manera: vivir con expectativas de cambio es una conexión con la realidad personal para elegir la vivencia de la salud, potenciar la interrelación persona-entorno, con fin de atreverse a nuevas posibilidades de vivir la salud. $\mathrm{La}$ estructura fue depurada aún más, al realizar la integración 


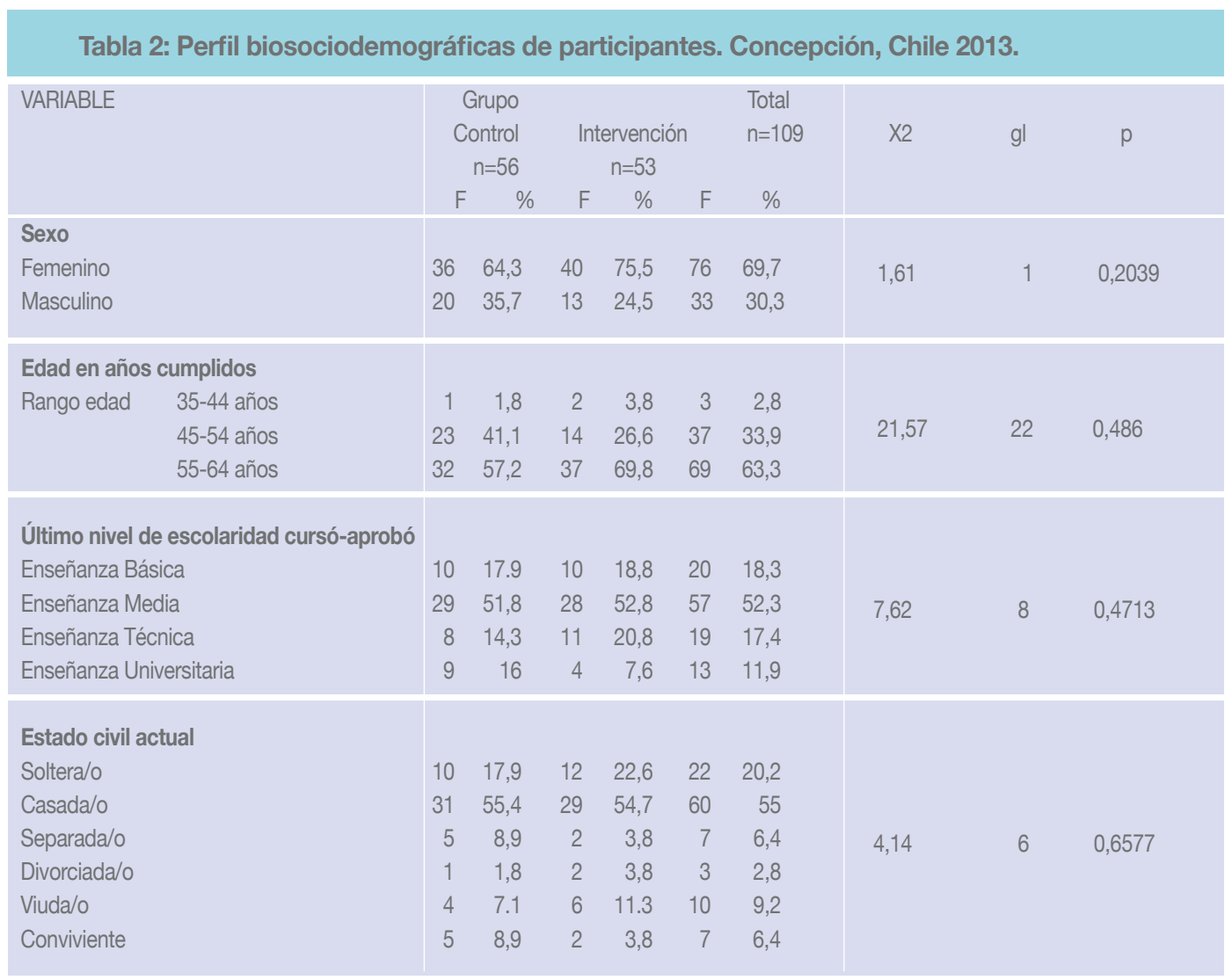

conceptual con la Teoría Humanbecoming: Vivir con expectativas de cambio es permitir-limitar, conectar-separar con el valor que se le otorgue a la vivencia de la salud, originando adaptación a la variación en las condiciones que se van presentando en el estado de salud y el poder personal para perseverar en las acciones que se proyectan en una imagen por medio del lenguaje.

\section{Discusión}

La intervención de consejería tuvo un efecto significativo en el aumento de la CVRS en las mujeres. Un ejemplo del efecto obtenido por este tipo de intervención se ilustra con una frase de una participante: "Me siento con más energía, alegre, ágil, con más vitalidad, más relajada y eso me hace dormir bien" (Marisol).

En cambio, en los hombres participantes los hallazgos encontrados en la CVRS no fueron significativos, lo que concuerda con lo reportado por un estudio sobre eficacia de un programa de intervención para el control de peso en personas con hipertensión, donde se observó que la CVRS mejoró en los integrantes del grupo intervención, pero sin diferencias significativas. ${ }^{20}$ Sin embargo, ellos reportaron: "Me siento con más dinamismo, más liviano, mejor ánimo" (Isidro). En las mujeres el peso y la CA disminuyeron significativamente y en los hombres solamente disminuyó la CA. Este efecto pudo haber sido motivado por la utilización del podómetro en la consejería, ya que se ha reportado que su uso en intervenciones en atención primaria conduce a un aumento en la actividad física de los usuarios aunado al apoyo social brindado por parte del profesional de la salud ${ }^{21}$. Esto se ve reforzado por la siguiente frase: "Yo ando por todas partes contando los pasos con mi instrumento (podómetro)...el material también es muy bonito y también los logros en estos meses" (Elaine). Los resultados obtenidos en los niveles de PA de las mujeres participantes evidencian la necesidad de 


\section{Tabla 3- Efecto de Intervención Innovadora: Consejería de Enfermería Personalizada y Telefónica en variables del estudio} de las mujeres del grupo control e intervención. Concepción, Chile 2013.

\begin{tabular}{|c|c|c|c|c|c|c|c|c|c|}
\hline \multirow{2}{*}{$\begin{array}{l}\text { MUJERES } \\
\text { Variables }\end{array}$} & \multicolumn{3}{|c|}{ Grupo Control } & \multicolumn{3}{|c|}{ Grupo Intervención } & \multirow{2}{*}{$\begin{array}{l}\text { Efecto de la Intervención } \\
\qquad(\text { IC95\%) }\end{array}$} & \multirow{2}{*}{ Estadígrafo } & \multirow{2}{*}{ Valor-p } \\
\hline & Pre-test & Pre-test & $\begin{array}{c}\text { Diferencia } \\
\text { pre y pos-test }\end{array}$ & Pre-test & Pre-test & $\begin{array}{c}\text { Diferencia } \\
\text { pre y pos-test }\end{array}$ & & & \\
\hline Col-Total & 178,2 & 176 & 2,22 & 201,1 & 178,7 & 22,4 & $-20,18(-36,42 ;-3,94)$ & $2,48^{\star}$ & $0,0156 \ddagger$ \\
\hline Col-HDL & 51,8 & 52,3 & $-0,5$ & 52,5 & 50,6 & 1,93 & $-2,43(-4,52 ;-0,33)$ & $2,31^{*}$ & $0,0237 \S$ \\
\hline Col-LDL & 105,3 & 104,1 & 1,19 & 123,4 & 103,1 & 20,24 & $-19,05(-33,48 ;-4,61)$ & $2,63^{\star}$ & $0,0104 \ddagger$ \\
\hline LDL/HDL & 2 & 2 & 0,04 & 126 & 2,4 & 2,1 & $-0,25(-0,5 ; 0,00)$ & $1,97^{*}$ & $0,0527 \S$ \\
\hline Triglicéridos & 105,7 & 98,1 & 7,67 & 126 & 124,7 & 1,23 & $6,44(-17,47 ; 30,35)$ & $1384 \dagger$ & 0,9834 \\
\hline TC/HDL & 3,5 & 3,4 & 0,09 & 3,9 & 0,7 & 0,35 & $-0,26(-0,56 ; 0,03)$ & $1260 †$ & 0,1899 \\
\hline $\begin{array}{l}\text { Riesgo Coronario } \\
\text { a10 años }\end{array}$ & 2,4 & 0,8 & 0,81 & 2,7 & 0,8 & 0,88 & $-0,07(-0,49 ; 0,35)$ & $1361,5 \dagger$ & 0,7848 \\
\hline Presión Sistólica & 129,2 & 133,4 & $-4,28$ & 133,2 & 131,6 & 1,63 & $-5,9(-12,16 ; 0,35)$ & $-1,88$ & 0,0641 \\
\hline Presión Diastólica & 89,6 & 77,1 & 12,47 & 91,5 & 78,9 & 12,63 & $-0,15(-5,14 ; 4,84)$ & $-0,06$ & 0,9515 \\
\hline Peso & 65,4 & 65,3 & 0,08 & 67,3 & 66,4 & 0,94 & $-0,85(-1,7 ;-0,01)$ & $1192 \dagger$ & $0,0435 \S$ \\
\hline $\begin{array}{l}\text { Circunferencia } \\
\text { Abdominal }\end{array}$ & 93,1 & 92,3 & 0,84 & 95,8 & 91,5 & 4,35 & $-3,51(-5,27 ;-1,75)$ & $1001,5 \dagger$ & $0,0001 \ddagger$ \\
\hline IMC & 27,6 & 27,6 & 0,05 & 28,1 & 27,7 & 0,38 & $-0,33(-0,69 ; 0,02)$ & $1204 \dagger$ & 0,0583 \\
\hline $\begin{array}{l}\text { Componente } \\
\text { Salud Física }\end{array}$ & 77,1 & 81 & $-3,93$ & 79,7 & 88,9 & $-9,2$ & $5,27(0,16 ; 10,38)$ & $2,06^{*}$ & $0,0433 \S$ \\
\hline $\begin{array}{l}\text { Componente } \\
\text { Salud Mental }\end{array}$ & 68,5 & 73,3 & $-4,82$ & 66,9 & 78,9 & $-11,95$ & $7,14(1,21 ; 13,06)$ & $2,40^{*}$ & $0,0189 \ddagger$ \\
\hline $\begin{array}{l}\text { Autoeficacia } \\
\text { General }\end{array}$ & 34,3 & 36,8 & $-2,5$ & 36 & 38,4 & $-2,37$ & $-0,13(-2,3 ; 2,05)$ & $0,11^{*}$ & 0,9091 \\
\hline $\begin{array}{l}\text { Apoyo Social } \\
\text { Percibido }\end{array}$ & 36,5 & 38,1 & $-1,58$ & 35,9 & 39,7 & $-3,8$ & $2,22(-6,5 ; 1,9)$ & $1472,5 \dagger$ & 0,3660 \\
\hline
\end{tabular}

*Test t para grupos pareados, †Test de Wilcoxon. $\ddagger p \leq 0,01, \S p \leq 0,05$

prolongar el tiempo de intervención de enfermería; ya que se ha reportado que después de 18 meses se logran cambios significativos en estas variables ${ }^{22}$. Los hombres disminuyeron significativamente la presión sistólica y diastólica, lo que concuerda con una intervención realizada durante 6 meses, dirigida a personas con exceso de peso e hipertensión ${ }^{20}$. Respecto al perfil lipídico, se obtuvo una disminución significativa como efecto atribuible a la intervención, en mujeres y hombres. Ello podría deberse a la incorporación de una alimentación saludable y aumento de actividad física. Las expresiones de las mujeres y hombres participantes lo ilustran: “...de repente yo comía mucha comida chatarra, los completos, papas fritas con mayo... ahora las hacía pero una vez a lo lejos, no tan seguido” (Luz); “...en este tiempo continúo con el régimen de mejorar la alimentación, de evitar todo lo que me ha perjudicado, y llevar el régimen que debería haber lleva- do y ahora lo estoy llevando, de comer cosas más sanas, verduras, legumbres" (Marvin). Se evidenció un efecto en la disminución del riesgo coronario a 10 años solo en los varones. Se concluye que esta intervención innovadora es una estrategia efectiva para el control de hipertensión, dislipidemia y sobrepeso y el mejoramiento de CVRS en el grupo intervención. El grupo control no presentó cambios significativos. En cuanto a lo cualitativo, el hallazgo fue la estructura de vivir con expectativas de cambio.

Entre las limitaciones del estudio se puede mencionar que los instrumentos utilizados no cuantificaron el apoyo social que brindó el profesional de enfermería ni la autoeficacia en el manejo de la enfermedad crónica. Además que no se estudiaron los factores de riesgo emergentes, como por ejemplo: concentraciones elevadas de apolipoproteína B, apolipoproteína A-1, disminución en la vitamina $\mathrm{D}$. 


\section{Tabla 4: Efecto de Intervención Innovadora: Consejería de Enfermería Personalizada y Telefónica en variables del estudio} en los hombres del grupo control e intervención. Concepción, Chile 2013.

\begin{tabular}{|c|c|c|c|c|c|c|c|c|c|}
\hline \multirow{2}{*}{$\begin{array}{l}\text { HOMBRES } \\
\text { Variables }\end{array}$} & \multicolumn{3}{|c|}{ Grupo Control } & \multicolumn{3}{|c|}{ Grupo Intervención } & \multirow{2}{*}{$\begin{array}{l}\text { Efecto de la Intervención } \\
\qquad(\mathrm{IC} 95 \%)\end{array}$} & \multirow{2}{*}{ Estadígrafo } & \multirow{2}{*}{ Valor-p } \\
\hline & Pre-test & Pre-test & $\begin{array}{c}\text { Diferencia } \\
\text { pre y pos-test }\end{array}$ & Pre-test & Pre-test & $\begin{array}{c}\text { Diferencia } \\
\text { pre y pos-test }\end{array}$ & & & \\
\hline Col-Total & 175,7 & 177,1 & $-1,4$ & 190,5 & 157,3 & 33,23 & $-34,63(-59,89 ;-9,38)$ & $2,80^{*}$ & $0,0088 \ddagger$ \\
\hline Col-HDL & 43,3 & 43,3 & 0 & 43,6 & 41,2 & 2,38 & $-2,38(-5,48 ; 0,71)$ & $1,57^{\star}$ & 0,1258 \\
\hline Col-LDL & 107,7 & 109,7 & $-2,03$ & 113,5 & 87,3 & 26,25 & $-28,28(-48,45 ;-8,11)$ & $2,86^{*}$ & $0,0075 \ddagger$ \\
\hline LDL/HDL & 2,5 & 2,6 & $-0,13$ & 2,6 & 2,1 & 0,49 & $-0,63(-1,05 ;-0,21)$ & $298 \dagger$ & $0,0045 \ddagger$ \\
\hline Triglicéridos & 123,6 & 120,9 & 2,65 & 167 & 144 & 23 & $-20,35(-59,02 ; 18,32)$ & $1,07^{*}$ & 0,2919 \\
\hline $\mathrm{TC} / \mathrm{HDL}$ & 4,1 & 4 & 0,05 & 4,4 & 3,8 & 0,54 & $-0,49(-0,94 ;-0,04)$ & $2,23^{*}$ & $0,0033 \S$ \\
\hline $\begin{array}{l}\text { Riesgo Coronario } \\
\text { a10 años }\end{array}$ & 3,5 & 3,2 & 0,3 & 4,7 & 3,1 & 1,62 & $-1,32(-2,6 ;-0,03)$ & $271,5 \dagger$ & $0,0546 \S$ \\
\hline Presión Sistólica & 135,1 & 139,7 & $-4,55$ & 141,9 & 132,2 & 9,77 & $-14,32(-24,78 ;-3,85)$ & $-2,79$ & $0,0089 \ddagger$ \\
\hline Presión Diastólica & 91,8 & 83,6 & $8,20^{*}$ & 99 & 81,1 & 17,92 & $-9,72(-18,00 ;-1,44)$ & $-2,4$ & $0,0228 \S$ \\
\hline Peso & 78,3 & 78,2 & 0,05 & 81,5 & 80,4 & 1,06 & $-1,02(-2,68 ; 0,65)$ & $244 \dagger$ & 0,3966 \\
\hline $\begin{array}{l}\text { Circunferencia } \\
\text { Abdominal }\end{array}$ & 96,6 & 96,8 & $-0,23$ & 101,5 & 97 & 4,50 & $-4,73(-7,03 ;-2,42)$ & $4,17^{*}$ & $0,0002 \ddagger$ \\
\hline IMC & 27,5 & 27,5 & 0,03 & 28,4 & 28,1 & 0,37 & $-0,34(-0,92 ; 0,25)$ & $243 \dagger$ & 0,4176 \\
\hline $\begin{array}{l}\text { Componente Salud } \\
\text { Física }\end{array}$ & 80,5 & 86,5 & $-6,03$ & 84,4 & 90,3 & $-5,85$ & $-0,18(-5,63 ; 5,27)$ & $0,07^{\star}$ & 0,9462 \\
\hline $\begin{array}{l}\text { Componente } \\
\text { Salud Mental }\end{array}$ & 72 & 77,3 & $-5,26$ & 78,7 & 81,3 & $-2,57$ & $-2,69(-8,63 ; 3,26)$ & $0,92^{*}$ & 0,3638 \\
\hline Autoeficacia General & 36 & 37,7 & $-1,65$ & 37,5 & 39 & $-1,46$ & $-0,19(-3,18 ; 2,8)$ & $238 \dagger$ & 0,5227 \\
\hline $\begin{array}{l}\text { Apoyo Social } \\
\text { Percibido }\end{array}$ & 37 & 38,7 & $-1,7$ & 38,7 & 41,7 & -3 & $1,3(-4,67 ; 7,27)$ & $0,44^{*}$ & 0,6602 \\
\hline
\end{tabular}

${ }^{*}$ Test t para grupos pareados, †Test de Wilcoxon. $\ddagger p \leq 0,01, \S p \leq 0,05$ 


\section{Referencias}

1. ORGANIZACIÓN MUNDIAL DE LA SALUD. Global status report on non-communicable diseases 2010. Geneva: WHO; 2011.

2. MINISTERIO DE SALUD, PONTIFICIA UNIVERSIDAD CATÓLICA DE CHILE, UNIVERSIDAD ALBERTO HURTADO. Encuesta Nacional de Salud ENS Chile 2009-2010. Santiago, Chile: Ministerio de Salud, Pontificia Universidad Católica de Chile; 2011.

3. JOVA R, RODRÍGUEZ A, DÍAZ A, BALCINDES S, SOSA I, DE VOS P, et al. Modelos de atención a pacientes con enfermedades crónicas no transmisibles en Cuba y el mundo. MEDISAN. 2011;15:1609-20.

4. ORGANIZACIÓN MUNDIAL DE LA SALUD. Informe sobre la salud en el mundo 2008. La atención primaria de salud más necesaria que nunca. Ginebra [Suiza]: OMS; 2008.

5. ORGANIZACIÓN MUNDIAL DE LA SALUD. Global status report on non-communicable diseases. Edición 2010. Geneva: WHO; 2011.

6. GRUPO AD HOC MERCOSUR. Primer Reporte de Vigilancia de Enfermedades No Transmisibles (ENT) 2011. Situación Epidemiológica de las ENT y Lesiones en Argentina, Brasil, Chile, Paraguay y Uruguay. Chile: Unidad de Estudios y Vigilancia de Enfermedades no Transmisibles del Departamento de Epidemiología del Ministerio de Salud de Chile; $2011.47 \mathrm{p}$

7. VALDÉS P. Innovaciones organizativas en atención primaria: líneas de futuro comunicación presentada en las XI Jornadas de
Medicina de Familia y Comunitaria; 14-15 de mayo de 2009; Vitoria-Gasteiz, España.

8. MORENO ME. Importancia de los modelos conceptuales y teorías de enfermería: experiencia de la Facultad de Enfermería de la Universidad de La Sabana. Aquichan. 2005; 5: 44-55.

9. PLUMMER M, MOLZAHH A. Quality of life in Contemporary Nursing Theory. A Concept Analysis. Nurs Sci Q. 2009; 22: $134-149$

10. CRESWELL J, PLANO V. Design and conducting mixed methods research. 2ed. Sage; 2011.

11. MCHORNEY C, WARE J, RACZEK A. The MOS 36-Item Short-Form Health Survey (SF-36): II. Psychometric and Clinical Tests of Validity in Measuring Physical and Mental Health. Med Care. 1993; 31: 247-63.

12. BÄßLER J, SCHWARZER R, JERUSALEM M. Spanish Adaptation of the General Self-Efficacy Scale. Auto-Eficacia Generalizada [Internet]. Berlín (AL): Freie Universität Berlin, Departamento de Psicología de la Salud; 1993 [actualizado 26 diciembre 1997; citado 20 mayo 2012]. Disponible en: http:// userpage.fu-berlin.de/ health/spanscal.htm

13. ZIMET G, DAHLEM N, ZIMET S, FARLEY G. The Multidimensional Scale of Perceived Social Support. J Pers Assess. 1988; 52: 30-41.

14. AMERICAN COLLEGE OF SPORTS MEDICINE. Editor Kaminsky L. Manual ACSM para la valoración del fitness relacionado con la salud. 4 ed. España: Wolters Kluwer Health; 2014. 
15. MINISTERIO DE SALUD. Guía clínica hipertensión arterial primaria o esencial en personas de 15 años y más. Santiago, Chile: Minsal, 2010.63 p.

16. PROGRAMA DE INVESTIGACIÓN FACTORES DE RIESGO DE ENFERMEDADES CARDIOVASCULARES. Función de riesgo coronario a 10 años adaptada a la población chilena [Internet]. Talca [Chile]: Facultad de Salud, Universidad de Talca; 2007 [actualizada 02 noviembre 2011, citada 03 de junio 2014]. Disponible en: http://pifrecv.utalca.cl/htm/riesgo_coronario/index.htm

17. PARSE R. The human becoming modes of inquiry: Emerging sciencing. Nurs Sci Q. 2005; 18: 297.

18. VÍLCHEZ V, PARAVIC T, SALAZAR A. La escuela de pensamiento Humanbecoming: una alternativa para la práctica de la enfermería. Cienc Enferm. 2013; 19: 23-34.

19. VÍLCHEZ-BARBOZA V. Efecto de intervención innovadora: consejería de enfermería en salud cardiovascular en atención primaria [Tesis Doctoral]. [Concepción]: Universidad de Concepción; 2014.

20. FERNÁNDEZ L, GUERRERO L, ANDUGAR J, CASAL M, CRESPO N, GUTIERREZ J, et al. Eficacia de un programa de intervención sobre control del peso en pacientes obesos hipertensos. Rev Soc Esp Enferm Nefrol. 2006; 9: 6-11.

21. HARRIS T, KERRY SM, VICTOR CR, SHAH SM, ILIFFE $\mathrm{S}$, USSHER M, et al. PACE-UP (Pedometer and consultation evaluation -UP) - a pedometer-based walking intervention with and without practice nurse support in primary care patients aged 45-75 years: study protocol for a randomised controlled trial. Trials. 2013: 14: 418-36.

22. CICOLINI G, SIMONETTI V, COMPARCINI D, CELIBERTI I, DI NICOLA M. et al. Efficacy of a nurse-led email reminder program for cardiovascular prevention risk reduction in hypertensive patients: A randomized controlled trial. Int J Nurs Stud. 2014; 51: 833-43. 